\title{
Low-Power Radio-Optical Beacons For In-View Recognition
}

\author{
Ashwin Ashok ${ }^{\dagger}$, Chenren $\mathrm{Xu}^{\dagger}$, Tam Vu${ }^{\ddagger}$, Marco Gruteser ${ }^{-1}$, Richard Howard ${ }^{-1}$, Yanyong Zhang ${ }^{-1}$ \\ Narayan Mandayam ${ }^{-1}$, Wenjia Yuan ${ }^{-1}$, Kristin Dana ${ }^{-1}$ \\ ${ }^{\dagger}$ Carnegie Mellon University, ${ }^{\ddagger}$ University of Colorado, Denver, ${ }^{-}$WINLAB, Rutgers University
}

\begin{abstract}
Object recognition on wearable devices using computer vision is too energy intensive and challenging when objects are similar looking, while low-power active radio frequency identification (RFID) systems suffer from imprecise orientation (angle and distance) estimates. To address this challenge, this paper presents a novel radio-optical based recognition system where a radio-optical transmitter, or tag, that emits a beacon whose infra-red (IR) signal strength is used for accurate relative orientation tracking of tagged objects at a wearable radio-optical receiver. A low-power radio link that conveys identity is used to reduce the battery drain by synchronizing the radio-optical transmitter and receiver so that extremely short optical pulses are sufficient for precise orientation estimation. Through extensive experiments with our prototype we show that our system can achieve orientation estimates with $1-2^{\circ}$ accuracy and within $40 \mathrm{~cm}$ ranging error, with a maximum range of $9 \mathrm{~m}$ in typical indoor use cases. With a tag battery power consumption of $86 \mu \mathrm{W}$, the radio-optical tags show potential to achieve about half a decade lifetimes.
\end{abstract}

\section{INTRODUCTION AND PRIOR ART}

The smart-glass concept [1], [2] provides endless possibilities for applications that can interact with the physical world. We identify that, in applications that interact with objects (or contexts) in the physical world, recognizing those objects that are within the user's view can be very important, apart from just knowing its absolute location. As illustrated in Fig. 1, the in-view recognition problem on smart-glasses translates to knowing the precise identity and relative orientation or position (of the user) to the object of interest.

Two key challenges prevail in realizing object recognition in smart-glasses: (i) automatic and precise identification of the subjects/objects in a person's view, and (ii) optimized battery usage. Earlier object recognition solutions can be broadly categorized into three approaches: (1) the positioning/tracking approach, (2) the computer vision approach, and (3) the tagging approach. Designs, so-far using these techniques, typically trade-off between accuracy and battery-lifetime. For example, the Wikitude World Browser [3] adopts the first approach by fusing GPS and compass signals, however, is limited to outdoors and does not fare well (in accuracy) when objects of interest are placed closely together. Computer vision approaches works best with known landmarks [4] or previously recognized subjects/objects [5]. The accuracy of this approach degrades, however, as lighting conditions deteriorate, the number of candidate objects/subjects becomes very large, or the objects themselves look very similar (e.g., boxes in a

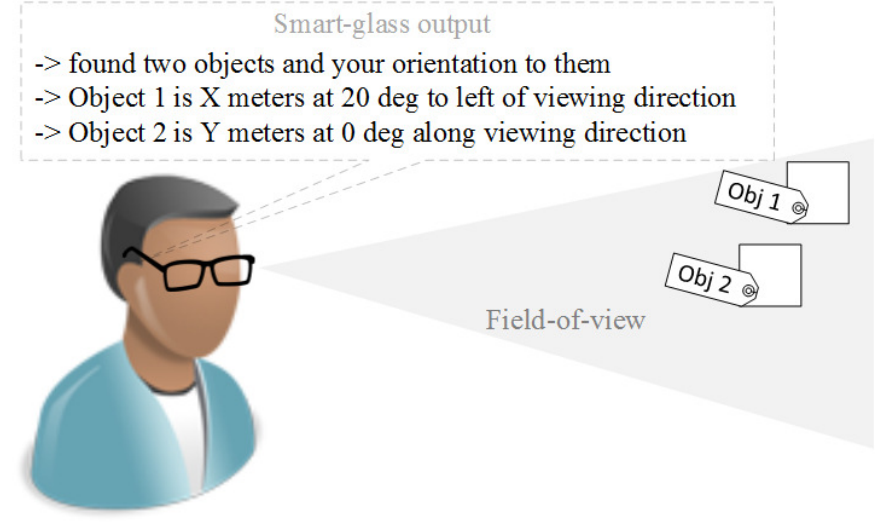

Fig. 1. Illustrating in-view recognition using smart-glasses

warehouse). Moreover, camera operations are energy intensive and usually not optimized for long-term usage on battery power. The third approach involves tagging objects of interest with a transmitting device, that emits signals which carry a unique identifying code pertaining to the object. An equivalent receiver that communicates with the transmitter recognizes the tagged object.

Precise positioning using radio signals has been achieved by existing tagging approaches using active RFID [6] (radiofrequency identification) or using passive reflections of radio signals [7] on array receivers. The Active Badge system [8] is an example of commercially deployed optical (infrared) tagging based positioning systems, but is very energy intensive; making it infeasible for applying to wearable systems. In general, existing techniques trade-off accuracy with energyconsumption or complexity. Therefore, designing a simple, precise yet low-power object recognition remains an open challenge.

A Hybrid Radio-Optical Beaconing Approach. We address the object recognition challenge by proposing a hybrid radiooptical system, that uses active radio frequency identification along with orientation tracking using near-IR (infrared) signals. We propose to use the tagging approach, however, with a novel tag and receiver design. The key component of our design is the radio-optical signal, or beacon as we will refer to, which is an ensemble of a RFID radio packet and an IR pulse. Infrared signals, due to their high directionality, can 


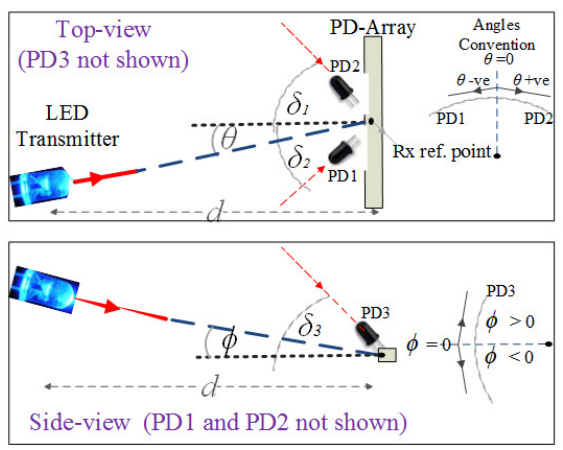

Fig. 2. Single LED transmitter - three element PD (photodetector) array receiver model $\left(\delta_{1}=\delta_{2}=\delta_{3}=\delta\right)$

lead to precise orientation tracking through angle-of-arrival (AoA) and distance estimation with a relatively small receiver, due to their small $850 \mathrm{~nm}$ wavelength. Unlike radio, they do not travel through visual obstructions and are less susceptible to multipath. The main advantage of our proposed hybrid approach is that it efficiently minimizes energy consumption by timing an infrared pulse using a RF side-channel. This enables the receiver to know when to expect the IR pulse and thus allowing for using extremely short IR pulses due to tight synchronization between the transmitter and receiver. In addition to reduced energy consumption, short IR pulses also lead to a simplified IR receiver design - instead of requiring an infrared communication receiver (such as in TV remote controls), a synchronized energy detection circuit suffices.

\section{Radio-Optical Beaconing System Design}

We propose that objects of interest are fit with our radiooptical tags that communicates with an equivalent radio-optical receiver fit on the smart-glass; as illustrated in Fig. 1. The task of recognition involves answering the following three questions: (a) when does the object transmit information, (b) what information does the object transmit, and (c) where (in user's view of 3D space) is the object. Our design proposes the use of a radio link to communicate the precise timing (answering (a)) and identity of the tagged object (answering (b)), and use of an IR link whose signal strength is used to determine orientation - AoA and distance (answering (c)). The radio-optical receiver recognizes the radio-optical tag by estimating the AoA and distance from the signal strength of the transmitted IR pulse and associates its orientation estimates with the tag identity transmitted through a radio link. The radio and IR links are ensured to be synchronized through our protocol design.

Before we describe the key characteristics of this design, we introduce to the reader key parameter definitions in our system, which includes AoA, distance (or range) and IR pulse width. Fig. 2 illustrates the IR link model in our design, where a three-element photodetector IR receiver samples IR signals from an IR LED on the radio-optical tag. We define AoA as the angle between the receiver surface normal and the vector connecting the transmitter and the depicted reference point in the center of the photodetector array; on the horizontal plane (azimuthal) as $\theta$ and vertical plane (polar) as $\phi$. The photodiodes are rotated by an angle $\delta$ from the surface normal such that the angle between the LED and the vector in direction of photodiodes 1,2 and 3 is $\theta-\delta$ and $\theta+\delta$, and $\phi+\delta$, respectively. We will use $\delta_{i r}$ to represent the width of the IR pulse and $d$ as the distance along the viewing axis between the tag and receiver. We present the derivation procedure for AoA $(\theta$ and $\phi)$ and distance $(d)$ from IR signal strength in the Appendix section. We will now describe the key features of our design and refer to their illustrations in Fig. 3 (a) and (b).

(i) Extremely short IR pulsing. IR requires high power to overcome the high background illumination and noise (artificial or solar) in this spectrum. We measure that the instantaneous power consumption of an off-the-shelf IR LED to transmit a single $1 \mathrm{sec}$ duration pulse to achieve a $10 \mathrm{~m}$ range is about $3 \mathrm{~W}$ - as opposed to RF signals that can achieve much higher range with orders of magnitude less power consumption. However, light signals provide the directionality that is important for positioning systems, which may not easily be achieved using radio signals; directional radio transmitters are bulky and their form-factor may not suit for wearable systems. We minimize the transmission period of the IR signal to the point where it can no longer be used for communication purposes but is still detectable for angle and distance estimation. Theoretically, a single short IR pulse with maximum peak power, like an optical strobe light, can be detected even at typical IR range $(10 \mathrm{~m})$ and at very low average energy consumption due to its extremely short duration. The challenge, however, lies for the receiver in detecting when such a signal was transmitted, thus requiring efficient synchronization between the transmitter and receiver.

(ii) Synchronization protocol. Our design proposes periodic transmissions of RF packets following a CSMA type communication protocol; the colliding RF packets being rejected at the receiver. Following the transmission of a RF packet, after a very short predetermined time-interval (known to the transmitter and receiver), an IR pulse is transmitted. The receiver uses the end of the radio packet reception as a reference to synchronize with the incoming IR pulse, and then samples the received signal from the IR signal receptors (photodiodes) over the expected pulse duration. It also takes an additional noise measurement after the pulse duration to calibrate for the ambient IR noise-floor. The IR pulse itself carries no bits of information-the target's information (e.g., its ID) is included in the preceding radio packet. By using the RF link to synchronize IR signal, we significantly cut down the system energy consumption compared to an IRonly object recognition system; which would typically require multiple IR pulses to communicate the payload, where each pulse is equivalent to carrying a single bit. Clearly, the probability of RF collisions at the receiver as the number of transmitters are scaled up. However, through intelligent dutycycling techniques and using additional context (RF signal strength) at the receiver, the relevant RF transmissions may 

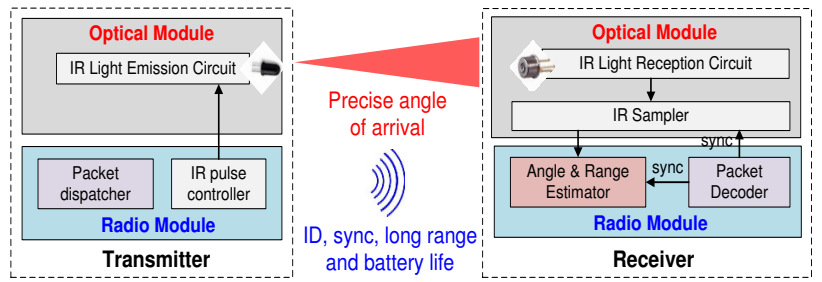

(a)

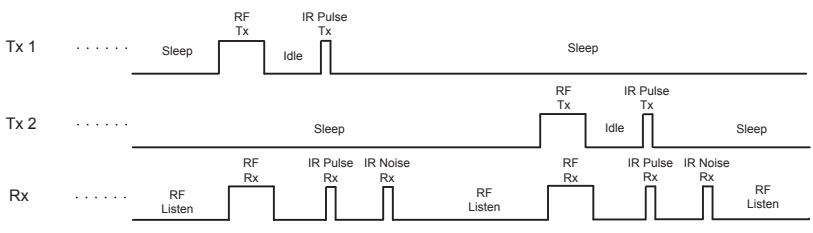

(b)

Fig. 3. (a) Radio-optical beaconing system architecture. The IR beacon is used for accurate positioning through AoA, while synchronization and ID communication is through radio, (b) Timing diagram of the paired-beaconing protocol over one duty-cycle period; this example uses 2 tags

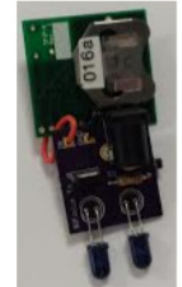

Transmitter

Tag

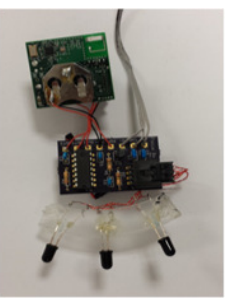

Receiver unit

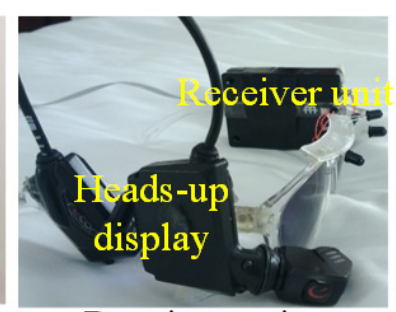

Receiver unit on

glasses
Fig. 4. Prototype tag and receiver (batteries not shown). The tag is $4 \mathrm{~cm}$ in largest dimension. Receiver unit is sized $(l \times w \times h)$ at $5 \mathrm{~cm} \times 4 \mathrm{~cm} \times 3 \mathrm{~cm}$.

be prioritized and/or filtered.

\section{Prototype Design}

We have prototyped the radio-optical tag, and a wearable receiver unit as shown in Fig. 4. We mounted the receiver unit on eye-glass together with a RECON Instruments MOD LIVE heads-up-display that runs Android. We developed a positioning application [10], where the tag orientations are estimated and displayed on the glass's heads-up display to help navigate the user to locate the tag in vicinity. We will now focus on the tag and receiver implementation.

Radio-Optical Tags. The transmitter tag consists of a RFID module that is used for the radio communication as well as triggering the pulse input to an IR LED. We used a MOSFET amplifier and an appropriate series resistor ensured that the current across each LED was maintained at $1 \mathrm{~A}$, for maximum emission. To maximize range, we used two near-IR LEDs [11] on the prototype tag powered by a $9 \mathrm{~V}$ battery supply. The $9 \mathrm{~V}$ power supply can be avoided by using a lower voltage battery along with a voltage step-up circuit. The RFID module on the tag contains a CC1100 radio (operating at $913 \mathrm{MHz}$ ) and a MSP430 microprocessor and powered by a CR2032 - 3V lithium coin cell battery. The radio operates at a data rate of $250 \mathrm{kbps}$ with MSK modulation and a programmed RF output power of $0 \mathrm{dbm}$. In each duty cycle, the radio broadcasts a 12-byte packet ( 4 bytes of preamble, 4 bytes of sync, 1 byte of packet length, 3 bytes of tag id + parity bits), waits for a short duration (measured to be at least $500 \mu$ s: over-the-air packet time of $380 \mu$ s and $120 \mu$ s hardware delay), triggers a
$3 \mathrm{~V}$ pulse for a duration of $\delta_{i r}=10 \mu$ s on one general purpose I/O pin connected to the MOSFET gate, and goes back to its sleep mode. The radio wakes up every $\tau=1 \mathrm{sec}$ and repeats the transmission.

Radio-Optical Receiver The front end of the receiver consists of three Silicon photodiodes [12]. Two of them are horizontally spaced by $3 \mathrm{~cm}$ and mounted with $40^{\circ}$ separation (at halfpower angle $\delta=20^{\circ}$, symmetrical on each side); the third is placed $20^{\circ}$ off (on top) the horizontal plane formed by the other two. With this setting, our receiver achieves an angularcoverage of $\pm 20^{\circ}$, and can be increased by placing more photodiodes in the receiver array. We amplify the detected IR signal through an opamp circuit with a rise-time much smaller than the IR pulse period. The receiver RFID module contains a CC1100 radio and a MSP430 microprocessor, similar to the radio-optical tag. Each photodiode's analog output from the opamp is wired to each of the three 12-bit analog-to-digital converter (ADC) input pins of the microprocessor. We power the radio using one of the $3 \mathrm{~V}$ supplies to the opamp (the opamp requires $\mathrm{a}+\mathrm{Vcc}$ and $-\mathrm{Vcc}$ supply). We programmed the radio to stay in an always-active-and-receive mode. Upon a successful packet reception the signal from the photodiodes are sampled at each ADC, and at a time instance after the end of packet reception - subject to a small hardware delay. The ADC sampling duration is set equal to the length of the IR pulse. The receiver identifies each tag through the unique transmit ID encoded in the radio packet.

\section{EXPERIMENTAL EVALUATION}

We conducted extensive experiments in a well-lit academic laboratory environment in different real-world application settings using our prototype tags and eye-glasses fit with the receiver. We evaluated the performance of our system based on the following metrics:

(1) Orientation estimation accuracy. The accuracy of determining the AoA and distance between the smart-glass on the user and a tag. We define this accuracy through the AoA and distance estimation error metrics, respectively.

(2) Power consumption: We evaluate the average battery power consumption of our tag and receiver units separately.

(a) Orientation Estimation Accuracy. We then conducted experiments to emulate real-world usage scenarios with our prototype system. We selected four scenarios, where 5 tags 


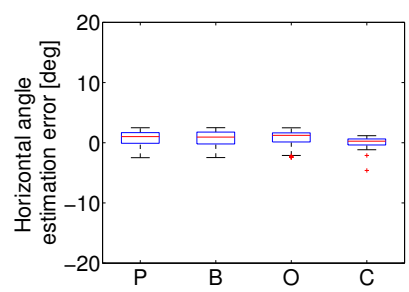

(a) Horizontal (b) Vertical

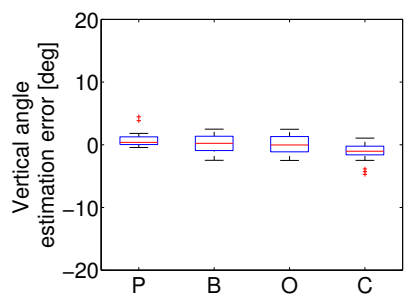

Fig. 5. Angle-of-arrival estimation error for the four application scenarios (P, B, O, C refer to Posters, Bookshelf, Office-room, Cubicle scenarios respectively)

each were fit onto (a) Posters, (b) Bookshelf racks, (c) objects in an Office-room and (d) books and devices on a cluttered Cubicle. Using calibrated ground truth measurements from a camera (with an IR lens), we observe that the median angular estimation errors was within $1.2^{\circ}$ (as shown in Fig. 5) and the ranging errors about $40 \mathrm{~cm}$.

We conducted experiments to validate our AoA and distance estimation accuracy in a tightly calibrated setting. In this set of experiments, we marked locations on the laboratory floor for ground-truth angle and distance measurements. The measurements spanned -10 to $10^{\circ}$ in $1^{\circ}$ spacings on the horizontal, vertical, and from $5 \mathrm{~m}$ to $9 \mathrm{~m}$ in $1 \mathrm{~m}$ steps. At each marked test points, we positioned the tag and collected 60 consecutive beacon samples. We then repeated the entire procedure 5 times, yielding a total of 300 samples per test point. We performed our evaluations for the tag beaconing period of $1 \mathrm{sec}$ and an IR pulse length of $500 \mu$ s to maximize range. In this experiment, the receiver glasses and transmitter were both positioned (fixed) on a crate at an equal height of $60 \mathrm{~cm}$ from the floor. Our results indicated a median angle error of $1.5^{\circ}$ and median distance error of $43 \mathrm{~cm}$.

(b) Power consumption. We compute the tag average power consumption as $P_{a v g}=\frac{E_{t o t}}{\tau}$, where $\tau$ is the beaconing (duty-cycle) period. The total energy consumption $E_{t o t}$ of the tag is the cumulative amount of energy consumed by the three modules: microprocessor, radio, and IR. Through oscilloscope measurements we determined the current draw (hence the energy) from the battery source in different states of operation- separately for the radio and IR modules as they are powered by independent battery sources. Our power measurements indicate that the radio module and IR module consume $32.457 \mu \mathrm{W}$ and $52.78 \mu \mathrm{W}$ respectively, for $10 \mu \mathrm{s}$ IR pulse and $1 \mathrm{sec}$ duty-cycling, and about $90 \mathrm{~mW}$ on the receiver. Theoretically this yields a tag lifetime of 9.854 years on a $9 \mathrm{~V}$ alkaline battery (520mAhrs capacity) and about 2 days for the receiver for continuous operation using an alkaline AA battery.

\section{RELATED WORK}

RFID. A rich body of work exists in the area of positioning using RF-based ranging [13], [14]. However, it has been shown that radio signal strength (RSS) based ranging is poor in high multipath environments [15] such as indoors. Kusy et al. [16] propose to address the multipath problem by using the doppler shift effects between a sensor and an anchor node, each transmitting at different frequencies. However, it requires a large number of sensor nodes, making it infeasible in many practical settings.

IR based positioning. Commonly used in robotics, where a robot is equipped with IR transceivers, IR is a well known candidate for positioning [17]-[20]. However, due to the modulation, synchronization and demodulation circuitry involved, the power consumption of IR communication systems will be greater than a simple light beaconing. The Active Badge system [8], Firefly [21] and OPTOTRACK are other examples of commercially deployed IR localization systems that use IR positioning, but are very energy intensive; making them infeasible for wearable systems.

Vision based systems. There are vision systems that are assisted by LED markers for robust identification [22], [23], however, such systems require extra processing for communication as simple energy detection does not suffice. Bytelight [9] proposes to provide indoor navigation to user's through visible light communication (VLC) from customized light bulbs. However, such customizations may not be possible in all applications. We refer readers to [24], for a rich list of different camera based localization techniques, for more details. In general, these approaches are limited in terms of range or require relatively large, costly and energy expensive cameras.

Augmented Reality (AR) Tagging: Prior work in positioning has implemented AR tagging in the form of $\mathrm{QR}$ codes and customized barcodes [25]-[28]. Raskar et al. [29] propose an augmented reality system where users are notified of objects fit with passive RFID tags equipped with photoreceptors detecting light from the user's projection device. However, mobile projectors, and cameras are costly as well as energy intensive, while pervasive use of barcodes in the physical world can be intrusive.

The need for a day is a light-weight, accurate and energy efficient solution for positioning. Our design takes a step ahead in this direction.

\section{CONCLUSIONS}

In this paper, we argued that a hybrid radio-optical beaconing approach can facilitate accurate and low-power recognition of objects within a user's view. Our approach leverages the high directionality characteristic of an infrared link for precise angle and distance estimation, and the low power nature of a radio link for synchronization and communication. The novelty of this design lies in the usage of a radio link to synchronize the infrared beacons such that very short highenergy infrared pulses could be used, which results in much reduced energy consumption, a simplified receiver design, and small hardware size. We prototyped the system by designing radio-optical tags and a wearable receiver, in the form of an object tracking eye-glasses. Our prototype receiver locates the infrared tags with an angular accuracy of $1.2^{\circ}$ on the horizontal and vertical dimensions, and up to $9 \mathrm{~m}$ distance at very low 
battery power consumption, supporting tag battery life of the order of years.

\section{APPENDIX}

The signal strength of an IR pulse of a predetermined duration $\delta_{i r}$ received by a photodetector, in terms of the photocurrent generated, can be expressed using the model from Kahn et al. [30] as,

$$
I_{p d}=\int_{\delta_{i r}} \frac{\gamma P_{l e d}(t)}{d^{2}} R_{p d}(\theta) R_{p d}(\phi) d t+\int_{\delta_{i r}} I_{n}(t) d t
$$

where $\gamma$ is a LED and photodetector specific constant (determined using datasheet); $P_{l e d}(t)$ denotes the LED irradiance (in $\mathrm{W} / \mathrm{sr}$ ) or the optical output power of the LED when it is $\mathrm{ON}$ at any time $t ; R_{p d}($.$) is the photodetector sensitivity$ function (normalized s.t. $R_{p d}(0)=1$ ). $I_{n}(t)$ denotes IR noise current at the receiver, typically dominated by shotnoise due to background light sources. The background noise current $\left(I_{n}\right)$ at any instance can be calibrated by measuring the received photocurrent when the LED is in the OFF state; that is, when $P_{l e d}(t)=0$, as the noise current changes very slowly with time [30].

AoA and distance estimation using IR Signal Strength. We will refer to Fig. 2 in this derivation. Let $I_{h 1}, I_{h 2}$, and $I_{v}$ represent the noise-subtracted IR signals $\left(I_{p d}-I_{n}\right.$, from equation (1)) on photodetectors 1, 2 and 3, respectively. We will consider that the photodetector sensitivity is lambertian $R_{p d}(x)=\cos ^{n}(x)$ where $n \geq 0$, typical for most off-the-shelf photodiodes available today and which can be obtained from the datasheet specifications. Based on equation 1, the noisesubtracted receiver photo-current generated due to the light energy accumulated from an LED emitting a constant light output $\left(P_{l e d}(t)\right.$ in equation 1 is a non-zero constant over the $\delta_{i r}$ duration, and zero otherwise) over a duration $\delta_{i r}$ varies proportionally. The derivation is completed as,

$$
\begin{gathered}
I_{h 1} \propto \gamma \cos ^{n}(\theta-\delta) \cos ^{n}(\Phi) \\
I_{h 2} \propto \gamma \cos ^{n}(\theta+\delta) \cos ^{n}(\Phi) \\
I_{v} \propto \gamma \cos ^{n}(\theta) \cos ^{n}(\Phi+\delta) \\
\theta= \pm \tan ^{-1}\left(\frac{1}{\tan (\delta)}\left|\frac{\left(\frac{I_{h 1}}{I_{h 2}}\right)^{1 / n}-1}{\left(\frac{I_{h 1}}{I_{h 2}}\right)^{1 / n}+1}\right|\right) \\
\Phi= \pm \tan ^{-1}\left(\cot \delta-2\left(\frac{I_{v}^{\frac{1}{n}}}{I_{h 1}^{\frac{1}{n}}+I_{h 2}^{\frac{1}{n}}}\right) \operatorname{cosec} \delta\right) \\
d=\left(\gamma \frac{\cos ^{n}\left(\theta_{e s t}+\delta\right)+\cos ^{n}\left(\theta_{e s t}-\delta\right)}{\left(I_{h 1}+I_{h 2}\right)}\right)^{\frac{1}{2}}
\end{gathered}
$$

where \pm indicate the directions relative to the reference point (left or right, up or down).

\section{REFERENCES}

[1] Google Glass. http://www.google.com/glass/start/.

[2] Meta. https://www.spaceglasses.com/.

[3] Wikitude : The world's leading augmented reality sdk. http://www.wikitude.com/, 2013.

[4] He Wang, Souvik Sen, Ahmed Elgohary, Moustafa Farid, Moustafa Youssef, and Romit Roy Choudhury. No need to war-drive: unsupervised indoor localization. In ACM MobiSys, 2012.

[5] Inside Microsoft Research: making purchases with zero effort. http://tinyurl.com/bkjt9js, 2013.

[6] Lei Yang, Yekui Chen, Xiang-Yang Li, Chaowei Xiao, Mo Li, and Yunhao Liu. Tagoram: Real-time tracking of mobile rfid tags to high precision using cots devices. In Proceedings of, MobiCom '14, pages 237-248, New York, NY, USA, 2014. ACM.

[7] Fadel Adib, Zachary Kabelac, Dina Katabi, and Robert C. Miller. 3d tracking via body radio reflections. In Proceedings of, NSDI'14, pages 317-329, Berkeley, CA, USA, 2014. USENIX Association.

[8] Roy Want, Andy Hopper, Veronica Falcão, and Jonathan Gibbons. The active badge location system. ACM Transactions on Information Systems, 1992.

[9] Bytelight : Indoor location. with light. http://www.bytelight.com/.

[10] Ashwin Ashok, Chenren Xu, Tam Vu, Marco Gruteser, Richard Howard, Yanyong Zhang, Narayan Mandayam, Wenjia Yuan, and Kristin Dana. Bifocus: Using radio-optical beacons for an augmented reality search application. In Proceeding of, MobiSys '13, pages 507-508, New York, NY, USA, 2013. ACM.

[11] TASL5300. www.vishay.com/docs/81008/tsal5300.pdf.

[12] BP10NF. http://www.vishay.com/docs/81503/bp10nf.pdf.

[13] P. Bahl and V.N. Padmanabhan. Radar: an in-building rf-based user location and tracking system. In IEEE INFOCOM, 2000.

[14] Kamin Whitehouse, Chris Karlof, and David Culler. A practical evaluation of radio signal strength for ranging-based localization. $A C M$ SIGMOBILE MCCR, 2007.

[15] Michael ODell, Regina ODell, Mirjam Wattenhofer, and Roger Wattenhofer. Lost in space or positioning in sensor networks. In REALWSN, 2005.

[16] Branislav Kusy, Akos Ledeczi, and Xenofon Koutsoukos. Tracking mobile nodes using rf doppler shifts. In ACM Conference on Embedded Networked Sensor Systems (SenSys), 2007.

[17] Larry Korba, Shadia Elgazzar, and Timothy Welch. Active infrared sensors for mobile robots. IEEE Transactions on Instrumentation and Measurement, 1994

[18] Eric Brassart, Claude Pegard, and Mustapha Mouaddib. Localization using infrared beacons. Robotica, 18(2):153-161, 2000.

[19] María V Moreno, Miguel A Zamora, José Santa, and Antonio F Skarmeta. An indoor localization mechanism based on rfid and ir data in ambient intelligent environments. In IEEE IMIS, 2012.

[20] Seongsu Lee and Sung-Yoon Jung. Location awareness using angle-ofarrival based circular-pd-array for visible light communication. In IEEE APCC, 2012.

[21] Firefly motion tracking system. http://www.gesturecentral.com/firefly/FireflyUserGuide.pdf, 1999.

[22] S. Hijikata, K. Terabayashi, and K. Umeda. A simple indoor selflocalization system using infrared leds. In IEEE INSS, 2009.

[23] News - casio unveils prototype of visible light communication system. bit.ly/zpfjY1.

[24] Rainer Mautz and Sebastian Tilch. Survey of optical indoor positioning systems. In IEEE IPIN, 2011.

[25] Artag. http://www.artag.net/, 2009.

[26] Ankit Mohan, Grace Woo, Shinsaku Hiura, Quinn Smithwick, and Ramesh Raskar. Bokode: imperceptible visual tags for camera based interaction from a distance. In ACM SIGGRAPH, 2009.

[27] Darnell J. Moore, Roy Want, Beverly L. Harrison, Anuj Gujar, and Ken Fishkin. Implementing phicons: combining computer vision with infrared technology for interactive physical icons. In ACM UIST, 1999.

[28] Hisashi Aoki and Soichiro Matsushita. Balloon tag: (in)visible marker which tells who's who. In ISWC, 2000.

[29] Ramesh Raskar, Paul Beardsley, Jeroen van Baar, Yao Wang, Paul Dietz, Johnny Lee, Darren Leigh, and Thomas Willwacher. Rfig lamps: interacting with a self-describing world via photosensing wireless tags and projectors. In ACM SIGGRAPH, 2004.

[30] J.M. Kahn and J.R. Barry. Wireless infrared communications. Proceedings of the IEEE, 1997. 